\title{
FISIOTERAPIA E ASSISTÊNCIA AO PARTO HUMANIZADO NO MUNICÍPIO DO RIO DE JANEIRO (RJ, BRASIL)
}

\author{
PHYSIOTHERAPY AND ASSISTANCE TO HUMANIZED DELIVERY \\ IN THE CITY OF RIO DE JANEIRO (RJ, BRAZIL)
}

Leidiane de Oliveira Santos (Orcid: 0000-0002-2095-3437) Juliana Veiga Cavalcanti (Orcid: 0000-0003-0469-4380) ${ }^{1}$

Karla Kristine Dames da Silva (Orcid: 0000-0002-6604-2823)

Michelle Guiot Mesquita (Orcid: 0000-0002-3361-467X)

Mauren Lopes de Carvalho (Orcid: 0000-0001-7994-9021)

\section{RESUMO}

Objetivo: identificar a oferta dos serviços públicos de assistência humanizada ao parto no município do Rio de Janeiro (RJ-Brasil), considerando a inserção do fisioterapeuta. Métodos: o mapeamento das instituições que realizam parto humanizado foi realizado por meio do Cadastro Nacional dos Estabelecimentos de Saúde (Cnes) e da literatura científica, com posterior visita em campo. O reconhecimento oficial da atuação fisioterapêutica nessa área foi verificado nos websites do conselho que fiscaliza a profissão e no Departamento de informática do Sistema Único de Saúde (Datasus). Resultados: doze instituições públicas realizam parto humanizado no município do Rio de Janeiro. Em apenas uma, há fisioterapeuta atuando diretamente na assistência ao parto, apesar do reconhecimento do conselho. Conclusão: o fisioterapeuta não tem sido aproveitado pelos serviços públicos no âmbito da assistência ao parto humanizado no município do Rio de Janeiro.

Palavras-chave: Fisioterapia; Trabalho de parto; Parto humanizado; Saúde da mulher.

\begin{abstract}
Objective: to identify the offer of humanized childbirth public assistance services in the city of Rio de Janeiro (RJ-Brazil), considering the integration of the physiotherapist professional. Method: the mapping of institutions that perform humanized childbirth was carried out by using the National Record of Healthcare Establishments (Cnes) and scientific literature, with subsequent field work. The official acknowledgement of the physiotherapeutic performance in this area was verified in the websites of the council that oversees the occupation and the Unified Health System IT Department (Datasus). Results: twelve public institutions that performed humanized childbirth in the city of Rio de Janeiro. Only one of them had a physiotherapist acting directly with childbirth assistance, despite the recognition of the council. Conclusion: the physiotherapist is not being properly used by public services in the context of humanized childbirth care in the city of Rio de Janeiro.

Keywords: Physical Therapy Specialty; Labor, Obstetric; Humanizing Delivery; Women's Health.
\end{abstract}




\section{INTRODUÇÃO}

No município do Rio de Janeiro, implantou-se a primeira política pública explícita de Humanização do Parto em 1990, adotando novas práticas e rotinas institucionais, como a implantação da assistência aos partos de baixo risco por profissionais não médicos ${ }^{1}$.

Tal ação ocorreu em alinhamento com a demanda do movimento organizado de mulheres, que, desde 1984, com o processo de redemocratização do país, reivindicava direitos relacionados com a igualdade, a sexualidade e a importância da assistência digna no período gestacional. Com a criação do Conselho Nacional dos Direitos da Mulher em 1985, foi organizado um encontro nacional em Brasília, que, em 1986, aprovou a Carta das Mulheres Brasileiras aos Constituintes, garantindo a assistência em toda a gestação ${ }^{2,3}$.

Em 1993, a constituição da Rede pela Humanização do Parto e Nascimento (Rehuna) ${ }^{4}$ foi fundamental para a estruturação do movimento ${ }^{5}$. Em 1995, foi lançado o Projeto Maternidade Segura, contendo um conjunto de parâmetros e procedimentos técnicos e avaliativos de serviços, denominado Oito Passos para a Maternidade Segura, fortalecendo as iniciativas de incentivo ao parto normal humanizado ${ }^{4}$. Tal projeto foi elaborado pelo Ministério da Saúde (MS), em parceria com a Federação Brasileira de Ginecologia e Obstetrícia (Febrasgo), o Fundo das Nações para a Infância (Unicef), a Organização Pan-Americana de Saúde (Opas), a Organização Mundial de Saúde (OMS) e o Fundo das Nações Unidas.

A partir de 2001, o MS criou manuais e políticas que abrangem a assistência humanizada do parto, como, por exemplo, o manual Parto, aborto e puerpério - Assistência Humanizada à Mulher ${ }^{6}$, a Política de Humanização do Parto e Nascimento do Brasil (PHPN), que é representada pelo Programa de Humanização no Pré-Natal e Nascimento e pelo Caderno HumanizaSUS - Humanização do Parto e Nascimento ${ }^{7,8}$.
Apesar de todos esses programas e políticas incentivadores do parto humanizado, a prevalência de cesáreas continua elevada no Brasil. Em 2015, foi divulgada a declaração da OMS sobre "epidemia de cesáreas no mundo, sendo o Brasil o líder". No município do Rio de Janeiro, segundo o Sistema de Informações sobre Nascidos Vivos ${ }^{10}$, dos 86.549 nascimentos no ano de 2012, 42,1\% foram partos vaginais; e $57,8 \%$, partos cesáreos, enquanto a OMS recomenda $15 \%$ de cesarianas ${ }^{11}$.

Preocupados com a alta prevalência de morte de gestantes e parturientes, as Nações Unidas lançaram, em 2015, uma Estratégia Global para a saúde de mulheres, crianças e adolescentes. Esta chama a atenção para a necessidade de oferecer assistência respeitosa, uma vez que experiências prévias de desrespeito se refletem em perda de confiança e inibem a busca das mulheres pela assistência nos serviços de saúde ${ }^{12}$.

Considerando as diferenças estruturais e fisiológicas do corpo feminino, além da particularidade da gestação e do parto, a fisioterapia foi considerada importante no tratamento conservador, assim, surgindo a nova área de fisioterapia na saúde da mulher. Esta inclui, além de avaliação, prevenção e promoção da saúde, condutas fisioterapêuticas nas alterações cinesiofuncionais do ciclo menstrual, climatério, disfunções urogenitais, constipação, incontinência urinária e fecal, prolapsos genitais, disfunções sexuais, preparação para a gestação e para o parto humanizado, acompanhamento do parto, pós-parto e oncológica ${ }^{13,14}$.

Corroborando o exposto, pesquisas afirmam que o acompanhamento na gestação por um fisioterapeuta bem como a assistência no trabalho de parto podem contribuir para a redução das taxas de cesáreas. A preparação para o parto desmistifica esse processo fisiológico natural, proporcionando confiança e preparando fisicamente o corpo da mulher ${ }^{15}$.

Atualmente, no Brasil, na Política da Estratégia Saúde da Família, ações de atenção básica à saúde das gestantes são 
acompanhadas em grupos por equipe interdisciplinar do Núcleo de Apoio à Saúde da Família (Nasf). Tais equipes têm em sua maioria a presença do fisioterapeuta, que atua na promoção da saúde mediante as alterações fisiológicas da gestação ${ }^{16}$.

Além disso, no trabalho de parto, a assistência fisioterapêutica reduz a dor e o sofrimento ${ }^{15}$. Segundo estudo de Domingues et al. ${ }^{17}$, a imagem de dor e sofrimento está fortemente associada ao trabalho de parto e ao parto. Assim, a fisioterapia contribui para o parto respeitoso e humanizado, como rege a OMS e a PHPN. Além disso, o conceito internacional de maternidade respeitosa leva em consideração, entre outros itens, a oferta de recursos para alívio da dor e a oportunidade de a mulher participar das decisões a respeito do seu processo de parto ${ }^{12}$. Por outro lado, no Brasil, a PHPN é desconhecida por muitas gestantes, que consideram o atendimento humanizado como sorte ${ }^{18,19}$.

Assim, o objetivo deste trabalho foi levantar a oferta dos serviços públicos de assistência humanizada ao parto no município do Rio de Janeiro, considerando a possibilidade de inserção do fisioterapeuta neste processo.

\section{MÉTODOS}

Trata-se de um estudo observacional, descritivo e transversal. Inicialmente, foi efetuado o levantamento do número de instituições que realizam parto no município do Rio de Janeiro e prestam serviço para o Sistema Único de Saúde (SUS). Esse levantamento foi realizado com base no Cadastro Nacional dos Estabelecimentos de Saúde (Cnes) e na dissertação "Avaliação da Política de Humanização ao Parto e Nascimento no município do Rio de Janeiro"4 em setembro de 2015.

Após a verificação desses dados, foi realizada aproximação ao campo, visitando as instituições identificadas entre outubro e novembro de 2015, a fim de confirmar a informação sobre a realização de parto humanizado no local. Tal informação foi fornecida pelas recepções e/ou centros de estudos das instituições.

As informações coletadas nessa primeira etapa foram utilizadas para a confecção de um mapa do município contendo a distribuição espacial das instituições que declararam realizar parto humanizado. Tais instituições foram selecionadas para etapas posteriores da pesquisa.

Ainda por meio do Cnes, foi possível identificar a presença de fisioterapeutas trabalhando nessas instituições. $\mathrm{Na}$ aproximação ao campo, essas informações foram confirmadas, bem como foi identificado o setor de atuação desse profissional, que poderia ou não atuar na assistência ao parto.

Entendendo a participação da fisioterapia na área de obstetrícia ainda como uma atividade emergente, buscouse, nos websites dos Conselhos Regionais de Fisioterapia (Crefito) e departamento de informática do SUS (Datasus) a presença dessa especialidade. O Crefito disponibiliza uma lista de especialidades da fisioterapia; e o Datasus, uma lista de ocupações de nível superior, baseada nas especialidades disponíveis pela Classificação Brasileira de Ocupação (CBO). O objetivo foi verificar o quanto que, pelo menos teoricamente, a presença da fisioterapia na assistência ao parto está prevista e assim identificar se há reconhecimento social desta atuação do fisioterapeuta.

O projeto foi aprovado pelo Comitê de Ética em Pesquisa do Instituo Federal do Rio de Janeiro, parecer $n^{\circ}$ 1.478 .579 .

\section{RESULTADOS}

Identificaram-se no Cnes e na leitura da dissertação de Boaretto ${ }^{4} 24$ instituições públicas que realizam parto no 


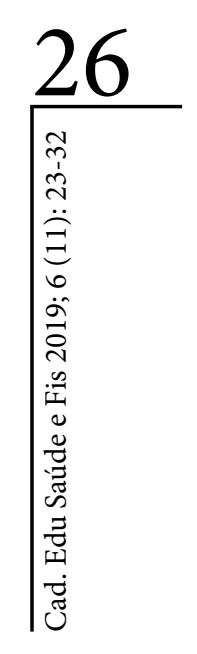

município do Rio de Janeiro. Após a aproximação ao campo, das 24 instituições, 12 informaram que realizam parto humanizado, 6 não realizam parto humanizado, 1 não informou e 5 foram extintas. $\mathrm{O}$ mapa com a distribuição dessas unidades revela imediatamente que elas estão mais concentradas nas regiões central e norte do município (Figura 1).

Segundo a Secretaria Municipal de Saúde - Rio (SMS - Rio) ${ }^{20}$, a cidade do Rio de Janeiro tem $1.224 \mathrm{~km}^{2}$ de área e densidade demográfica de 5.163 habitantes $/ \mathrm{km}^{2}$, organizada em 33 regiões administrativas e 160 bairros, apresentando-se bastante heterogênea, com diferentes graus de desenvolvimento e, consequentemente, desigualdade na distribuição e utilização dos recursos disponíveis, inclusive dos serviços de saúde. Para fins de plane- jamento em saúde, a SMS - Rio trabalha, desde 1993, com a divisão da cidade em dez áreas de Planejamento: 1.0, 2.1, 2.2, 3.1, 3.2, 3.3. 4.0, 5.1, 5.2 e 5.3 .

Considerando essa informação, as instituições que realizam parto humanizado estão localizadas nas áreas programáticas 1.0, 2.1, 2.2, 3.2, 3.3. 4.0, $5.1 \mathrm{e}$ 5.2 (Quadro 1).

Das 12 unidades que realizam parto humanizado, segundo dados do Cnes, quatro possuem fisioterapeutas gerais no seu quadro de funcionários, e uma não contém fisioterapeutas. Nas demais sete unidades, após aproximação ao campo, foi identificada uma que contém fisioterapeuta atuando diretamente no parto e fisioterapeutas gerais, três que contêm fisioterapeutas atuando no pré-natal,

Figura 1. Mapeamento das instituições que realizam parto no monucípio do Rio de Janeiro em outubro/novembro de 2015

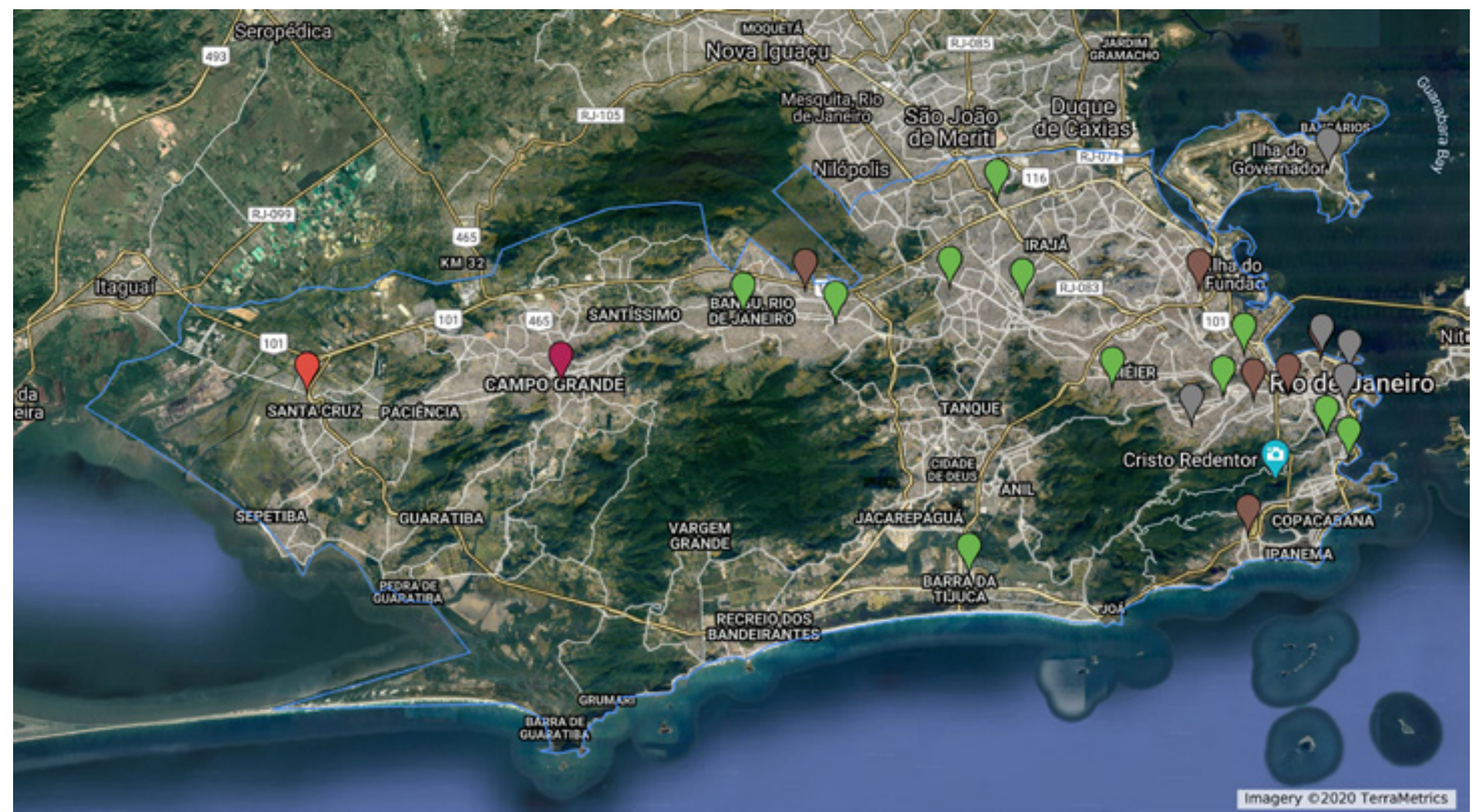

Legenda: em cinza - instituições extintas; marrom - instituições que não realizam parto humanizado; verde - instituições que realizam parto humanizado; vermelho - instituição que não forneceu informação. 
Quadro 1. Áreas programáticas onde estão localizadas as Instituições que realizam Parto Humanizado pelo SUS no município do Rio de Janeiro e fisioterapeutas inseridos no quadro de funcionários destas instituições em 2015

\begin{tabular}{|c|c|c|}
\hline Área Programática & $\begin{array}{l}\text { No de insti- } \\
\text { tuições que } \\
\text { realizam parto } \\
\text { humanizado }\end{array}$ & Fisioterapeutas no quadro de funcionários \\
\hline 1.0 & 1 & $\begin{array}{l}\text { - Fisioterapeuta atuando no pré-natal, pós-parto ou neonatal e fisiote- } \\
\text { rapeutas gerais }\end{array}$ \\
\hline 2.1 & 2 & $\begin{array}{l}\text { - Fisioterapeuta atuando no pré-natal, pós-parto ou neonatal } \\
\text { - Fisioterapeuta atuando no pré-natal, pós-parto ou neonatal e fisiote- } \\
\text { rapeutas gerais }\end{array}$ \\
\hline 2.2 & 1 & $\begin{array}{l}\text { - Fisioterapeuta atuando no pré-natal, pós-parto ou neonatal e fisiote- } \\
\text { rapeutas gerais }\end{array}$ \\
\hline 3.2 & 1 & - 5 fisioterapeutas gerais \\
\hline 3.3 & 3 & $\begin{array}{l}\text { - } 18 \text { fisioterapeutas gerais } \\
\text { - } \quad \text { Fisioterapeuta atuando no pré-natal, pós-parto ou neonatal } \\
\text { - } \quad \text { Fisioterapeuta atuando no pré-natal, pós-parto ou neonatal }\end{array}$ \\
\hline 4.0 & 1 & - 12 fisioterapeutas gerais \\
\hline 5.1 & 2 & $\begin{array}{l}\text { - } 14 \text { fisioterapeutas gerais } \\
\text { - } \quad \text { Não contêm fisioterapeuta }\end{array}$ \\
\hline 5.2 & 1 & - 1 fisioterapeuta atuando diretamente no parto e fisioterapeutas gerais \\
\hline
\end{tabular}

Fonte: CNES e trabalho de campo entre outubro e novembro de 2015.

pós-parto ou neonatal e fisioterapeutas gerais e três que contêm apenas fisioterapeutas atuando no pré-natal, pós-parto ou neonatal (Quadro 1).

Quanto à existência das especialidades que contemplam a atuação fisioterapêutica no momento do trabalho de parto e parto, estas são denominadas Fisioterapia em saúde da mulher ou Fisioterapia urogineco-funcional. Estas foram identificadas na maioria dos websites dos conselhos regionais de fisioterapia no país que disponibilizam informações sobre as especialidades da profissão (Quadro 2).

No entanto, nas CBO relacionadas com a profissão do fisioterapeuta listadas no Datasus, dados do município do Rio de Janeiro na categoria Ocupações de nível superior, não há a categoria Fisio- terapia em saúde da mulher ou Fisioterapia urogineco-funcional, embora existam outras especialidades da fisioterapia, como fisioterapeuta traumato-ortopédico, fisioterapeuta respiratório, fisioterapeuta do trabalho etc. (Quadro 3).

\section{DISCUSSÃO}

Nossos resultados mostram que, no município do Rio de Janeiro, a maioria dos estabelecimentos onde há assistência ao parto normal, há a preocupação em realizar o parto humanizado. Isso demonstra que, até certo ponto, a demanda das mulheres por uma assistência ao parto mais digna vem sendo acolhida. Mesmo assim, ainda existe área programática não 


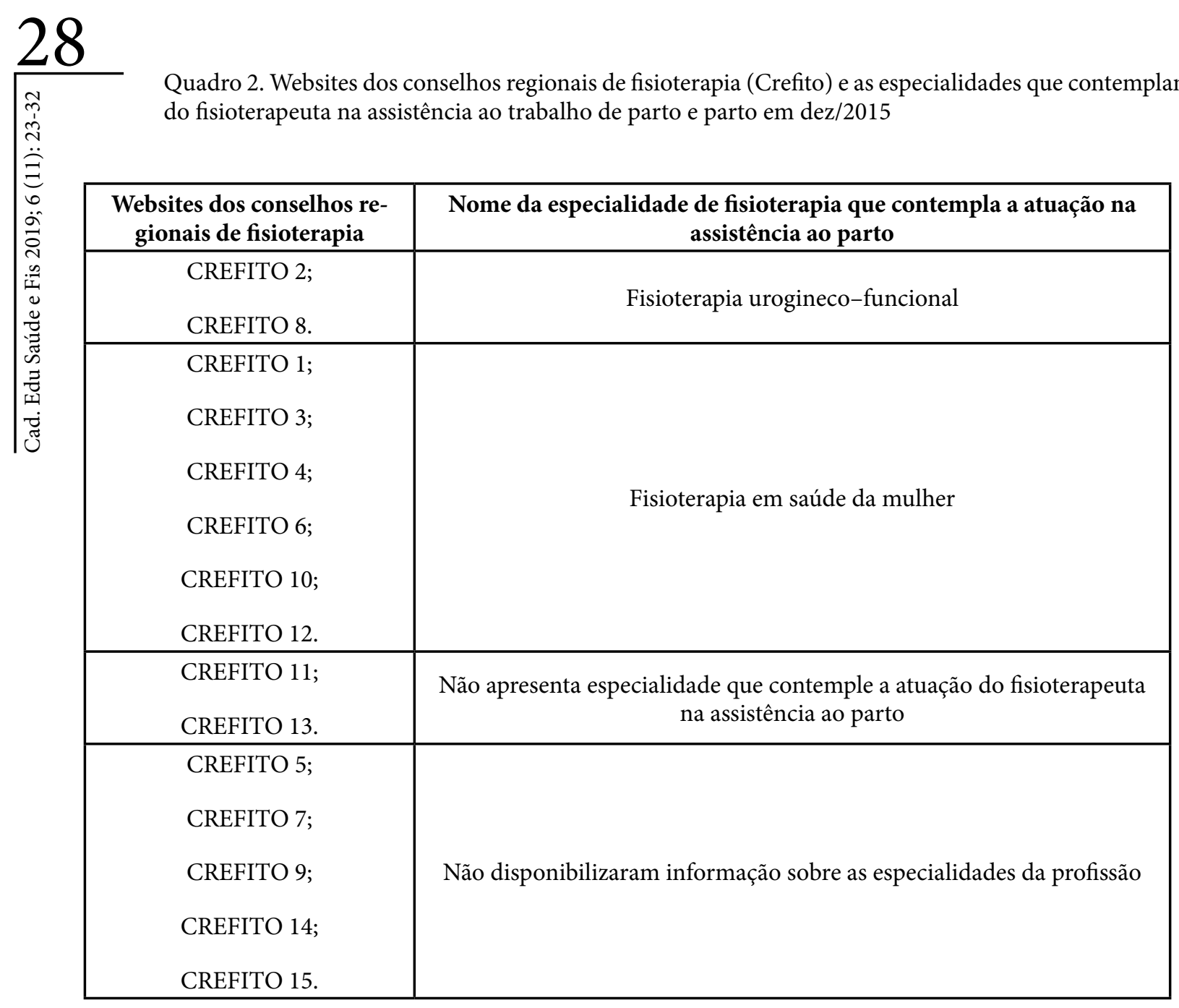

Fonte: Websites dos conselhos regionais de fisioterapia (Crefito), consulta realizada em 2015.

Quadro 3. Especialidades da fisioterapia e número de profissionais listados no Datasus em dez/2015 no município do Rio de Janeiro

\begin{tabular}{|l|c|}
\hline \multicolumn{1}{|c|}{ Ocupações de nível superior segundo o Datasus } & No de Profissionais no Rio de Janeiro \\
\hline Fisioterapeuta geral & 2202 \\
\hline Fisioterapeuta traumato-ortopédico & 40 \\
\hline Fisioterapeuta acupunturista & 40 \\
\hline Fisioterapeuta respiratório & 20 \\
\hline Fisioterapeuta neurofuncional & 8 \\
\hline Fisioterapeuta do trabalho & 7 \\
\hline Fisioterapeuta esportivo & 5 \\
\hline Fisioterapeuta quiropraxista & 1 \\
\hline Fisioterapeuta osteopata & 0 \\
\hline Outros profissionais & 0 \\
\hline
\end{tabular}

Fonte: Datasus em dez/2015. 
contemplada por esse tipo de assistência. Isso pode ocorrer devido a alguns desafios do modelo de assistência humanizada, entre eles, a mudança na cultura hospitalar, as modificações na estrutura física proporcionando ambientes acolhedores, profissionais que respeitem a fisiologia desse momento e a garantia da autonomia da mulher ${ }^{1}$.

Dias e Domingues ${ }^{1}$ ressaltam também a importância de profissionais que auxiliam para minimizar o sofrimento da parturiente, tornando esse momento uma experiência de crescimento e realização para a mulher e para sua família. Acreditando em uma nova abordagem que estimule a participação ativa da mulher e seu/sua acompanhante, que preconize o suporte físico e emocional e o uso de novas técnicas de cuidado que permitam o alívio da dor ${ }^{1}$.

Os desafios são muitos, apontados pelos mesmos autores em relato no ano de 2005 sobre a implantação na prática da política de humanização da assistência hospitalar em unidades de saúde do Rio de Janeiro. Apesar das evidências científicas mais diversas para que sejam realizadas modificações no modelo médico tradicional de assistência ao parto, desmedicalizá-lo implica a perda de poder; e o abandono desse Modus Operandi significa perda de controle do processo da parturição e das referências do papel do médico neste contexto. Para os autores, a proposta de humanização da assistência ao parto sofre influência direta da missão institucional, do modelo organizacional, do envolvimento e aderência dos gerentes à proposta, da capacitação e sensibilidade dos profissionais de diversos seguimentos da saúde. Entretanto, destacam que sua implantação efetiva estará sempre atrelada à relação entre a mulher e o profissional de saúde, sujeita aos inúmeros aspectos de suas subjetividades ${ }^{1}$.

Com relação ao reconhecimento formal da atuação do fisioterapeuta na assistência ao parto, o conselho que regulamenta a profissão reconhece formalmente essa atividade desde 2009, quando publica a Resolução Coffito 365, de 20 de maio de $2009^{21}$. Por outro lado, a CBO não possui uma categoria específica para o fisioterapeuta que atua na assistência ao parto, ficando este agregado à categoria fisioterapeutas gerais.

O mesmo ocorreu com a especialidade fisioterapia do trabalho. Após criarem a Associação Brasileira de Fisioterapia do Trabalho (Abrafit) e ser reconhecida pelo Conselho Federal de Fisioterapia e Terapia Ocupacional (Coffito), o passo seguinte para o reconhecimento dessa especialidade foi a de que o Ministério do Trabalho, pela CBO, descrevesse para o mercado brasileiro quem é esse especialista, especificando e detalhando suas práticas comprovadas nessa área, distinguindo áreas de atividade, competências pessoais e recursos de trabalho. Com isso, as empresas puderam realizar contratos de trabalho direcionados a essa especialidade/especialista ${ }^{22}$. Acredita-se, dessa maneira, que a ausência da especialidade de fisioterapia urogineco-funcional na $\mathrm{CBO}$ restringe o campo de atuação na área ao passo que tal reconhecimento facilitaria a realização de contratos de fisioterapeutas especializados na assistência ao parto, além das questões culturais destacadas anteriormente.

Bavaresco et al. $^{23}$, ao discorrerem sobre o papel do fisioterapeuta como profissional de suporte à parturiente, afirmam que a presença do fisioterapeuta no acompanhamento do parto, de maneira geral, não se estabeleceu ainda de maneira adequada em nossa sociedade. Entretanto, os autores colocam que esse profissional deve ter como premissa a orientação e a conscientização das mulheres, tornando-as mais seguras para o momento do parto. Além disso, afirmam que a intervenção fisioterapêutica na assistência obstétrica, como parte da rotina da equipe, surge como alternativa para a valorização da responsabilidade da gestante no processo, por meio do uso ativo do próprio corpo. 
A interação de fatores fisiológicos, psicológicos, culturais e o suporte da equipe profissional obstétrica influenciam na mobilidade corporal durante o processo de parturição. Dessa maneira, a atuação do fisioterapeuta pode ser um potencial fator de conscientização para a mulher em relação ao seu corpo ativo como potente ferramenta facilitadora do trabalho de parto, trazendo satisfação com a experiência do nascimento ${ }^{23}$.

Nesse contexto, faz-se uma analogia à vivência do parto humanizado, trazido por Santos e Okasaki ${ }^{24}$, quando colocam que a humanização da assistência ao parto necessita, especialmente, que os profissionais envolvidos respeitem os aspectos da fisiologia da parturiente, não intervindo sem necessidade no momento do parto, identificando os aspectos sociais e culturais do parto e do nascimento, agindo com atenção no suporte emocional da mulher e da sua família, facilitando a formação dos laços afetivos familiares e o vínculo entre a mãe e o bebê. Outros aspectos relevantes referem-se à autonomia da mulher durante todo o processo, com respeito ao planejamento do parto por parte dos profissionais, incluindo a escolha do acompanhante, procedimentos e outros fatores que envolvem sua vontade e direitos como cidadã.

Um estudo de Sodré et al. ${ }^{25}$ abordou a participação da mulher na tomada de decisão, nas ações de promoção à saúde, como um direito que deve ser respeitado no planejamento do parto. Esse estudo teve como objetivo compreender a necessidade de cuidado e o desejo de participação nas decisões sobre o parto de gestantes da rede pública e privada em Londrina. Ao analisarem os depoimentos das gestantes, os autores evidenciaram que, apesar de desejarem participar do planejamento do seu parto e de verbalizarem suas necessidades, escolhas e preferências, as mulheres não encontram condições favoráveis para que suas necessidades de cuidado e o desejo de participação nas decisões fossem viabilizados em ambos os cenários, tanto público quanto privado.
Os mesmos autores colocam que, das resistências encontradas pelas gestantes, observou-se a insensibilidade dos profissionais às suas necessidades; as condições do sistema de saúde; a falta de informações; a insegurança e o medo revelados; e os sonhos não atingidos, que acabaram produzindo frustrações nas mulheres. Dessa maneira, sob a ótica da humanização do cuidado, faz-se necessário o reconhecimento à individualidade da mulher e a identificação de suas necessidades, gerando relações menos autoritárias por parte dos profissionais e com maior autonomia da mulher, como condutora do processo $^{25}$.

A partir da perspectiva de Dias e Domingues 1 quanto ao perfil profissional, o fisioterapeuta apresenta competência para atuar na assistência ao parto humanizado. Outros trabalhos descrevem a importância do fisioterapeuta também no uso de técnicas aplicadas à parturiente de baixo risco para proporcionar conforto, alívio da dor, relaxamento e confiança em relação ao próprio corpo. Algumas estratégias incluem o estímulo à deambulação precoce, adoção de posturas verticais, exercícios respiratórios e de analgesia, massagens, banhos quentes, crioterapia e relaxamento, que, no contexto do parto humanizado, podem ser úteis para minimizar a sensação de desconforto, trazendo naturalidade para o momento da concepção ${ }^{26-29}$.

Freitas et al..$^{30}$ colocam que, segundo a OMS, faz-se necessária a revisão de técnicas apropriadas para o parto com ênfase à movimentação da parturiente, oferecendo liberdade para mudanças e livre escolha de posições no momento das contrações e trabalho de parto. Os autores colocam que a cinesioterapia, como recurso fisioterapêutico, pode ser uma potente ferramenta no auxílio para manter as parturientes ativas durante todo o período do trabalho de parto, por meio de uma redescoberta das posturas verticais sentada, ajoelhada, de cócoras e em quatro apoios, dando liberdade de movimento como prática eficiente que facilita o processo de 
parto a elas. Essa estratégia envolve a atuação do fisioterapeuta, sendo considerada de baixo custo, boa reprodutibilidade e fácil adesão. Outras estratégias, como as de controle da dor e relaxamento, são apontadas pelos autores como importantes na abordagem fisioterapêutica com objetivo de acatar os preceitos do parto humanizado. Embora esses benefícios sejam mais amplamente discutidos na literatura atualmente, ainda há um longo caminho até a efetiva inserção do fisioterapeuta e seus recursos na atenção às parturientes.

\section{CONCLUSÕES}

O presente estudo foi realizado a fim de identificar e analisar a oferta dos serviços de fisioterapia durante o trabalho de parto no município do Rio de Janeiro, considerando a PHPN. A maioria das instituições públicas que realizam parto normal nesse município está comprometida com a oferta de um serviço humanizado. Porém, das 24 instituições, apenas uma apresenta fisioterapeuta atuando diretamente neste modelo de parto. Isso ocorre mesmo com o reconhecimento da atuação pelo conselho que regulamenta a profissão.

A atuação fisioterapêutica no trabalho de parto e no parto está ainda em processo de reconhecimento. A CBO não apresenta uma categoria que contemple essa atuação; consequentemente, não há profissionais cadastrados no Datasus, o que afeta a oferta desse serviço que contribuiria para a assistência no modelo humanizado.

A fisioterapia urogineco-funcional ou fisioterapia em saúde da mulher apresenta um campo a ser explorado e ampliado na assistência ao parto, haja vista todos os benefícios das práticas fisioterapêuticas aplicadas apresentados aqui.

Este foi um estudo exploratório que pretendeu identificar a oferta dos serviços públicos de assistência humanizada ao parto no município do Rio de Janeiro, identificando a presença ou ausência do fisioterapeuta e, portanto, de seus recursos nesse processo. A contradição entre a existência de tais recursos e a ausência de profissionais atuando de fato nessa área deverá ser explorada por pesquisas qualitativas que possam explicar os entraves e sugerir novos caminhos para a maior participação do fisioterapeuta na assistência ao parto humanizado.

\section{REFERÊNCIAS}

1. Dias MAB, Domingues RMSM. Desafios Na Implantação de Uma Política de Humanização Da Assistência Hospitalar Ao Parto. Ciên Saúde Colet. 2005; 10(3): 669-705.

2. Coelho LM, Baptista MM. A História Da Inserção Política Da Mulher No Brasil: Uma Trajetória Do Espaço Privado Ao Público. Rev Psicol Polit. 2009; 9(17): 85-99.

3. Silva SM. A carta que elas escreveram: a participação das mulheres no processo de elaboração da constituição federal de 1988 [Dissertação]. Salvador (BA): Universidade Federal da Bahia, 2011.

4. Boaretto MC. Avaliação Da Política de Humanização Ao Parto e Nascimento no Município do Rio de Janeiro [Dissertação]. Rio de Janeiro (RJ): Escola Nacional de Saúde Pública/ Fiocruz; 2003.

5. Rehuna - Rede Pela Humanização do Parto e Nascimento. Quem Somos. [Internet], s.d. [Citado 11 abr 2015]; Disponível em: http://www.rehuna.org. br/index.php/quem-somos.

6. Brasil. Ministério da Saúde. Manual Parto, Aborto e Puerpério - Assistência Humanizada à Mulher. Brasília, DF: Ministério da Saúde; 2001.

7. Brasil. Ministério da Saúde. Programa de Humanização no Pré-natal e Nascimento. Brasília, DF: Ministério da Saúde; 2002.

8. Brasil. Ministério da Saúde. Caderno HumanizaSUS - Humanização do Parto e do Nascimento. Brasília DF: Ministério da Saúde; 2014.

9. UNA-SUS. Declaração da OMS sobre Taxas de Cesáreas. [Internet], 2015. [Citado 09 mai 2015]; Disponível em: http://www.unasus.gov.br/noticia/ declaracao-da-oms-sobre-taxas-de- cesareas.

10. Sinasc. TabNet Win32 3.1: Nascidos Vivos - Rio de Janeiro. [Internet], 2012. [Citado 12 abr 2015]; Disponível em: http://tabnet.datasus.gov.br/cgi/tabcgi.exe?sinasc/cnv/nvrj.def. 
11. Portal Brasil. Governo anuncia medidas para reduzir cesarianas desnecessárias. [Internet], 2014 [Citado 11 abr 2015]; Disponível em: http://www. brasil.gov.br/saude/2014/10/governo-anuncia-medidas-para-reduzir-cesarianas-desnecessarias.

12. Shakibazadeh E, Namadian M, Bohren MA, Voge JP, Rashidian A, Pileggi VN, et al. Respectful care during childbirth in health facilities globally: a qualitative evidence synthesis. An International Journal of Obstetrics \& Gynaecology. 2017; 125: 932-942.

13. Latorre GFS. Fisioterapia na Saúde da Mulher Florianópolis: CREFITO 10 [Internet]; 2010 [Citado em 09 de julho de 2010]. Disponível em: http://www. crefito10.org.br/conteudo.jsp?idc=393.

14. Conselho Federal de Fisioterapia e Terapia Ocupacional. Resolução $\mathrm{n}^{\circ} 401$ de 18 de agosto de 2011 . Disciplina a especialidade profissional de fisioterapia na saúde da mulher e suas providências. Diário Oficial da União. Brasília, DF: em 24 de novembro de 2011

15. Baracho E. Fisioterapia aplicada à saúde da mul her. $5^{\text {a }}$ Edição. São Paulo: Editora Guanabara Koogan; 2012

16. Brasil. Ministério da Saúde. Caderno de Atenção Básica - Diretrizes do NASF: Núcleo de Apoio à Saúde da Família. Brasília, DF: Ministério da Saúde; 2009.

17. Domingues RMSM, Santos EM, Leal MC. Aspectos da satisfação das mulheres com a assistência ao parto: contribuição para o debate. Cad Saúd Púb. 2004; 20(1): 52-62.

18. Dias MAB, Deslandes SF. Expectativas sobre a assistência ao parto de mulheres usuárias de uma maternidade pública do Rio de Janeiro, Brasil: os desafios de uma política pública de humanização da assistência. Cad Saúd Púb. 2006; 22(12): 2647-2655.

19. Van Der Spank JR, Cambier DC, De Paepe HMC Danneels LA, Wityrouw EE, Beeren L. Pain relief in laubour by transcutaneous electrical nerve stimulation (TENS). Arch Gynecol Obstet. 2000; 264: 131136.

20. Brasil. Secretária Municipal de Saúde do Rio de Janeiro. Plano Municipal de Saúde do Rio de Janeiro (PMS) 2014-2017. [Internet], 2013. [Citado 29 nov 2016]; Disponível em: http://www.rio.rj.gov.br/dlstatic/10112/3700816/4128745/PMS_20142017.pdf
21. Conselho Federal de Fisioterapia E Terapia Ocupacional. Resolução COFFITO 365, de 20 de maio de 2009. Reconhece a Saúde da Mulher como especialidade do profissional Fisioterapeuta e dá outras providências. Diário Oficial da União $n^{\circ}$. 112, Seção 1 página 42. Brasília, DF: em 16 de junho de 2009.

22. Baú LM, Klein AA. [Editorial]. O reconhecimento da especialidade em fisioterapia do trabalho pelo COFFITO e Ministério do Trabalho/CBO: uma conquista para a fisioterapia e a saúde do trabalhador. Rev Bras Fisiot. 2009; 13(2): 5-6.

23. Bavaresco GZ, Souza RSO, Almeica B, Sabatino JH, Dias M. O fisioterapeuta como profissional de suporte à parturiente. Ciên Saúde Colet. 2011; 16(7) 3259-3266

24. Santos IS, Okazaki ELFJ. Assistência de enfermagem ao parto humanizado. Rev Enferm UNISA. 2012; 13(1): 64-68

25. Sodré TM, Bonadio IC, Jesus MCP, Merigui MAB. Necessidade de cuidado e desejo de participação no parto de gestantes residentes em Londrina - Paraná. Texto Contexto Enferm. 2010; 19(3): 452-60.

26. Davim RMB, Torres GV, Melo ES. Estratégias não farmacológicas no alívio da dor durante o trabalho de parto: Pré-teste de um instrumento [Internet]. São Paulo: Rev. Latino-am. Enferm.; 2007. [Citado Nov./Dez. 2007 http://dx.doi.org/10.1590/ S0104-11692007000600015 ]. Disponível em: http://www.scielo.br/scielo.php?script=sci_arttext\& pid=S0104-11692007000600015\&lng=en\&tlng=en

27. Mamede FV, Almeida AN, Clapis MJ. Movimentação/deambulação no trabalho de parto: Uma revisão. Acta Scientarium. Health Science (Maringá). 2004; 26(2):295-302

28. Sartori AL, Vieira F, Almeida NAM, Bezerra ALQ, Martins CA. Estratégias não farmacológicas de alívio à dor durante o parto. Revista Electroníca trimestral de Enfermaría. 2011;21:1-9.

29. Nunes GS, Moreira PCS, Vial DSV. Recurso fisioterapêuticos para alívio da dor no trabalho de parto. Revista FAIPE. 2015; 5(1): 90-99.

30. Freitas AS, Lima VS, Sousa JN, Zuchelo LTS Martinelli PM. Atuação da fisioterapia no parto humanizado. DêCiência em foco. 2017; 1(1): 18-29. 